\title{
Aquaporin 4 over-expression in metastases to the brain in breast cancer.
}

\author{
Shahan Mamoor ${ }^{1}$, MS \\ 1Thomas Jefferson School of Law \\ San Diego, CA 92901 \\ mamoorsk@tjsl.edu
}

Brain metastases affect $10-15 \%$ of women with breast cancer (1). Metastasis is the most significant contributor to death in patients with cancer (2). We assessed what genes make brain metastases most different from the breast tumors from which they arose using public datasets $(3,4)$. The aquaporin 4 (AQP4) water channel (5) was one of the most differentially expressed genes in brain metastases when comparing the transcriptomes of matched tumor and metastasis samples from the brain and breast from 16 patients (2). Analysis of a separate dataset showed demonstrated the same result (4). In both cases, aquaporin 4 was expressed at significantly higher levels in metastases to the brain than in the primary breast tumor. This is the first report of aquaporin 4 differential over-expression in the brain metastases of patients with breast cancer.

Keywords: breast cancer, brain metastases, systems oncology, tumor transcriptome analysis, global differential gene expression profiling, aquaporin 4, AQP4. 


\section{Introduction}

Breast cancer is a leading killer of women in the United States and one of the leading cancers in women, second only to lung cancer (5). In 2017, over a quarter of a million cases of invasive breast cancer were reported in the United States and there were 40,610 deaths from breast cancer (5). Metastasis, or spread of the cancer to a foreign site such as the lung, bones or brain, is the major reason why humans expire from cancer (2). $10-15 \%$ of the women that develop breast cancer will develop brain metastases (1). There are no targeted treatments for women suffering from brain metastases caused by breast cancer. To understand the basic transcriptional nature of brain metastases, we utilized two datasets to compare brain metastases to primary breast tumors. In both datasets, we found that aquaporin 4 (AQP4) was one of the most differentially expressed genes in the brain metastasis transcriptome. AQP4 has functions relevant to the brain including transport of water (6). The brain-specific function of AQP4 makes it a promising therapeutic target, first for validation in larger cohorts, and second for assessment in functional assays in vivo and in vitro to determine if AQP4 supports or is required for survival or progression of brain metastases in breast cancer.

\section{Methods}

GSE125989 (3) and GSE100534 (4) was utilized for this analysis in conjunction with GEO2R. Investigators that created GSE125989 used Affymetrix Human Genome U133A 2.0 microarray technology. In GSE125989, $\mathrm{n}=16$ brain metastasis tissue samples and $\mathrm{n}=16$ primary breast tumor samples. These samples were matched (from the same 16 patients). In GSE100534, $n=16$ primary breast tumor samples and $n=3$ brain metastasis tissue samples. Investigators that created GSE100534 used Affymetrix Human Gene 1.0 ST Array. In this study, the Benjamin and Hochberg method of p-value adjustment was utilized, and logtransformation of data was set to "Auto-detect". The raw p-value is utilized here. The NCBI generated category of platform annotation was utilized. A statistical test was performed to evaluate the significance of difference in RNA expression values between each differentially expressed gene in primary breast tumors and brain metastases by a two-tailed, unpaired t-test 
with Welch's correction using PRISM (8.1.2) (227). Regardless of whether differences in exact mRNA expression levels are considered statistically significant, all differentially expressed genes described here are determined to be statistically significant with respect to global differential expression.

\section{Results}

We used global differential gene expression profiling of two public datasets $(3,4)$ to compare the transcriptomes of primary tumors of the breast and brain metastases from patients with breast cancer to understand in an unbiased fashion and at systems-level the most significant differences in gene expression between brain metastases in breast cancer and tumors of the breast. We found that the water channel aquaporin 4 was among the genes whose expression was most different between primary breast tumors and metastases to the brain.

Aquaporin 4 was identified as the 24th most differentially expressed gene out of 22277 total transcripts detected in the Iwamoto dataset (3) (Table 1; $\mathrm{p}=3.64 \mathrm{E}-06)$. Aquaporin 4 was expressed at significantly higher levels in brain metastases than in the breast tumors from the same patients (matched samples) (Figure 1; $p<0.0001$ ).

Aquaporin 4 was identified as the 86th most differentially expressed gene out of 33297 total transcripts detected in the Schulten dataset (4) (Table 1; $p=1.73 \mathrm{E}-06)$. In this second dataset, AQP4 was also expressed at higher levels in brain metastases than in the primary breast tumors to which they were compared to, but this difference in RNA expression level was not statistically significant (Figure $2 ; \mathrm{p}=0.1004$ ).

\section{Discussion}

In this study, we identified increased expression of aquaporin 4 in the brain metastases of patients with breast cancer by comparing the transcriptomes of brain metastases to matched primary tumor samples (3) and primary breast tumor samples from other patients (4). Aquaporin 4 was not only expressed at increased levels in brain metastases, but among the 
top $1 \%$ of differentially expressed genes when considering all of the genes in the transcriptome, meaning between breast cancers and the brain metastases they generate, the change in expression of aquaporin 4 was greater than $99 \%$ of the genes in the transcriptome.

Aquaporin 4 expression in the brain is widespread and is enriched in the phospholipid membranes of astrocytes, particularly at the blood-brain barrier (6). Aquaporin 4 is highly selective for the transport of water unlike other aquaporins which can also transport glycerol and other molecules (6). Mice that are deficient in aquaporin 4 (AQP4) exhibit enhanced survival after experiencing brain edema from acute water intoxication (7). The authors argued that AQP4 played a critical role in modulation of water transport in the brain (7). Arguing against the fact that differential aquaporin 4 expression in brain metastases is reflective of brain-specific gene expression rather than brain metastasis-specific gene expression is the fact that AQP4 is expressed at higher levels in edematous tumors of the brain than in benign, unaffected brain tissue (8).

The brain has an exceptionally high metabolic rate of oxygen (9). It composes only $2 \%$ of the total body weight of a human but consumes $20 \%$ of the oxygen (9). The only study that evaluated AQP4 function in oxygen gas exchange documented increased oxygen deprivation in micro-watershed areas of the brains of AQP4-deficient mice (10), suggesting that AQP4 loss caused a reduction in oxygen diffusion. Thus, it is possible that an increase in AQP4 expression permits brain metastases to fuel their oxygen metabolism by increasing uptake of gaseous oxygen.

We identified globally significant differential expression of aquaporin 4 in the brain metastases of patients with breast cancer when compared to primary tumors of the breast using two independent public datasets $(3,4)$. Aquaporin 4 has vital functions in the brain involving transport of water at junctions of the blood-brain barrier $(6,7)$. These results should be validated at the protein level in separate and larger cohorts and the effect of aquaporin 4 inhibition by shRNA knockdown in xenograft mouse models of brain metastatic breast cancer 
or of haploinsufficiency of aquaporin 4 should be evaluated, both with respect to time until metastasis, metastasis burden, and time until expiration. 


\section{References}

1. Rostami, R., Mittal, S., Rostami, P., Tavassoli, F. and Jabbari, B., 2016. Brain metastasis in breast cancer: a comprehensive literature review. Journal of neuro-oncology, 127(3), pp. 407-414.

2. Mehlen, Patrick, and Alain Puisieux. "Metastasis: a question of life or death." Nature Reviews Cancer 6.6 (2006): 449.

3. Iwamoto, T., Niikura, N., Ogiya, R., Yasojima, H., Watanabe, K.I., Kanbayashi, C., Tsuneizumi, M., Matsui, A., Fujisawa, T., Iwasa, T. and Shien, T., 2019. Distinct gene expression profiles between primary breast cancers and brain metastases from pairmatched samples. Scientific reports, 9(1), pp.1-8.

4. Schulten, H.J., Bangash, M., Karim, S., Dallol, A., Hussein, D., Merdad, A., Al-Thoubaity, F.K., Al-Maghrabi, J., Jamal, A., Al-Ghamdi, F. and Choudhry, H., 2017. Comprehensive molecular biomarker identification in breast cancer brain metastases. Journal of translational medicine, 15(1), p.269.

5. American Cancer Society. "Breast cancer facts \& figures 2017-2018." (2017)

6. Nagelhus, E.A. and Ottersen, O.P., 2013. Physiological roles of aquaporin-4 in brain. Physiological reviews, 93(4), pp.1543-1562.

7. Manley, G.T., Fujimura, M., Ma, T., Noshita, N., Filiz, F., Bollen, A.W., Chan, P. and Verkman, A.S., 2000. Aquaporin-4 deletion in mice reduces brain edema after acute water intoxication and ischemic stroke. Nature medicine, 6(2), pp.159-163.

8. Saadoun, S., Papadopoulos, M.C., Davies, D.C., Krishna, S. and Bell, B.A., 2002. Aquaporin-4 expression is increased in oedematous human brain tumours. J Neurol Neurosurg Psychiatry, 72(2), pp.262-265.

9. Lassen, N.A., 1959. Cerebral blood flow and oxygen consumption in man. Physiological reviews, 39(2), pp.183-238. 
Table 1-1

\begin{tabular}{|c|c|c|c|c|c|c|}
\hline Rank (dataset) & ID & p-value & $\mathbf{t}$ & B & Gene & Gene name \\
\hline \multicolumn{7}{|l|}{ (GSE125989) } \\
\hline 24 & 210066_s_at & 3.64E-06 & 5.419117 & 4.2602 & AQP4 & aquaporin 4 \\
\hline \multicolumn{7}{|l|}{ (GSE100534) } \\
\hline 86 & 8022655 & 1.73E-06 & 6.6805926 & 5.267525 & AQP4 & aquaporin 4 \\
\hline
\end{tabular}

Table 1: Aquaporin 4 is among the most differentially expressed genes when comparing the transcriptomes of primary breast tumors and brain metastases from humans.

The rank of differential expression with respect to 22277 (3) or 33297 (4) total transcripts, Affymetrix probe or transcript ID, $p$-value, t, a moderated t statistic, $B$, the log-odds of differential expression between the two groups, log-fold change of gene expression, the gene symbol and gene name are shown in the table above. 

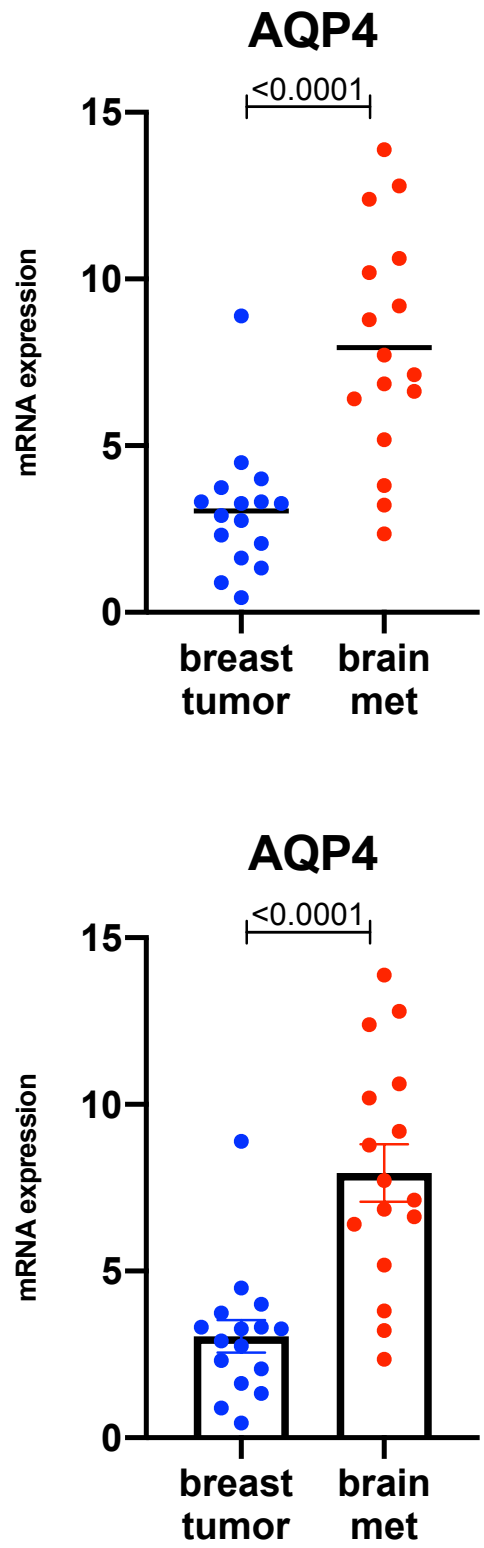

Figure 1: Aquaporin 4 is expressed at significantly higher levels in the brain metastases of patients with breast cancer when compared to their primary tumors.

The expression of AQP4 is graphically represented as dots denoting the expression level in each individual patient (above), and as a bar graph with the error bars denoting the standard error of the mean (below), with breast tumor tissue in blue and brain metastasis tissue in red. A statistical test was performed to evaluate the significance of difference in AQP4 expression between breast tumor and brain metastasis, and the results of this test is shown on each graph as a p-value. 

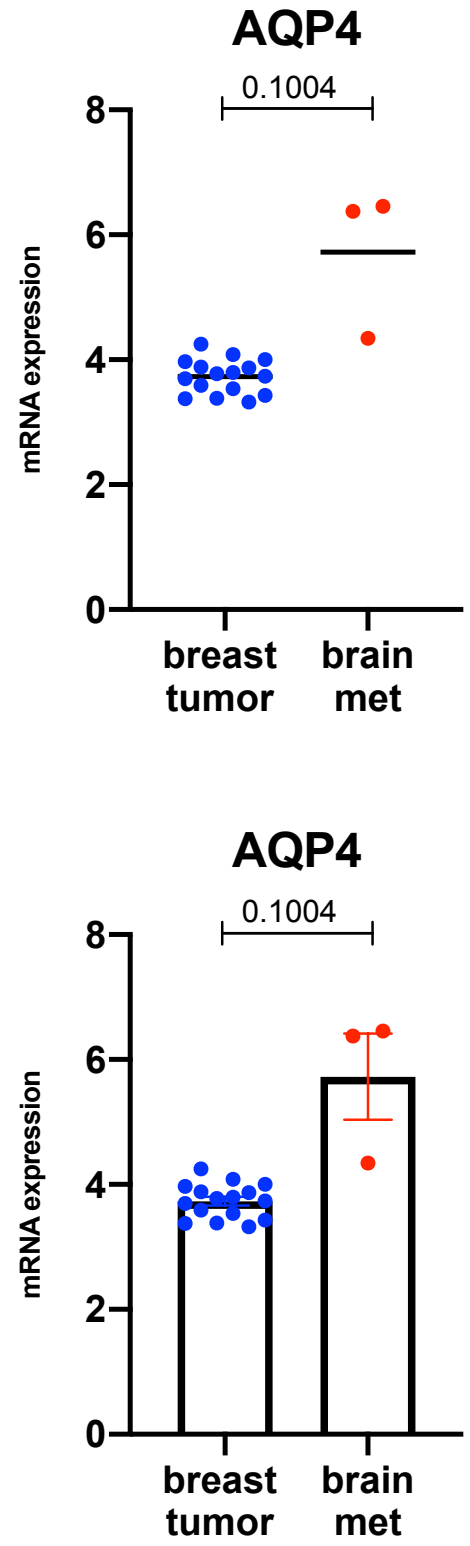

Figure 2: Aquaporin 4 is expressed at higher levels in the brain metastases of patients with breast cancer when compared to non-matched primary tumors of the breast.

The expression of AQP4 is graphically represented as dots denoting the expression level in each individual patient (above), and as a bar graph with the error bars denoting the standard error of the mean (below), with breast tumor tissue in blue and brain metastasis tissue in red. A statistical test was performed to evaluate the significance of difference in AQP4 expression between breast tumor and brain metastasis, and the results of this test is shown on each graph as a p-value. 